\title{
Camu camu (Myrciaria dubia) como posible alternativa productiva
}

\section{Camu camu (Myrciaria dubia) as a possible agricultural production}

\section{alternative}

\author{
Ardila Ortiz Leonardo ${ }^{1}$ y Yunda Constanza ${ }^{2}$ \\ ${ }^{1}$ Ingeniero Agrónomo, Universidad de los Llanos y \\ ${ }^{2}$ Ingeniera Agrónoma, MSc, Docente Universidad de los Llanos \\ cyunda@unillanos.edu.co
}

Recibido 11 de Agosto 2017, Aceptado 19 de Octubre 2017

\section{RESUMEN}

En este artículo se presentan los aspectos técnicos-agronómicos del camu camu (Myrciaria dubia), y su ecología, además se analiza el potencial que tiene esta especie como cultivo comercial, teniendo en cuenta la demanda agroecológica de la especie y las exigencias agronómicas para establecerla como sistema productivo en condiciones del piedemonte llanero, puesto que Colombia tiene gran diversidad de frutas exóticas con alto contenido nutricional, especies nativas como la mencionada han sido consideradas y priorizadas como alternativas en investigaciones de CORPOICA como una de las frutas tropicales con alto potencial para el desarrollo agrícola, aunque en Colombia solo se registra en los departamentos de Amazonas y Vaupés, por esta razón es importante revisar la información generada sobre la especie y analizar el potencial de ésta como sistema productivo para las condiciones del piedemonte llanero. La especie es importante por su alto valor nutritivo, puesto que el fruto del camu camu tiene el más alto nivel de vitamina $\mathrm{C}$ conocido hasta ahora, superando en aproximadamente 1.5 veces a la acerola (Malpighiae marginata) $(1790 \mathrm{mg} / 100 \mathrm{~g}$ ), en 13 veces al marañon (Anacardeum occidentale) $(219 \mathrm{mg} / 100 \mathrm{~g}$ ) y en 5 veces al limón (Citrus limon) (44,2 mg/100 g), en comparación con la naranja (Citrus sinensis) contiene 30 veces más vitamina $C, 10$ veces más hierro, 3 veces más niacina, 2 veces más riboflavina y $50 \%$ más fósforo.

Palabras clave: Fruto, vitaminas, cosecha, árboles, alimentos. 


\section{ABSTRACT}

This article presents the technical-agronomic aspects of the camu camu (Myrciaria dubia), and its ecology, in addition, the potential of this species as a commercial crop is analyzed, taking into account the agroecological demand of the species and the agronomic requirements to establish it as a productive system under the conditions of the foothills of the Plains, because Colombia has a great diversity of exotic fruits with a high nutritional content, native species such as the one mentioned have been considered and prioritized as alternatives in CORPOICA investigations as one of the tropical fruits with high potential for agricultural development, although in Colombia it only registers in the departments of Amazonas and Vaupés, for this reason it is important to review the information generated about the species and analyze the potential of this as a productive system for the conditions of the foothills of the Plains. The species is important for its high nutritional value, surpassing in approximately 1.5 times to acerola (Malpighiae marginata) (1790 mg/100 g), 3 times to marañon (Anacardeum occidentale) (219 mg/100 g) and 5 times to lemon (Citrus limon) $(44.2 \mathrm{mg} / 100 \mathrm{~g})$, compared to orange (Citrus sinensis) contains 30 times more vitamin C, 10 times more iron, 3 times more niacin, 2 times more riboflavin and $50 \%$ more phosphorus.

Keywords: Fruit, vitamins, harvest, trees, food.

\section{RESUMO}

Este artigo apresenta os aspectos técnico-agronômico do camu camu (Myrciaria dubia), e sua ecologia, além disso, o potencial desta espécie como cultura comercial é analisado, tendo em conta a demanda agroecológica das espécies e os requisitos agronômicos para estabelecê-lo como um sistema produtivo sob as condições dos contrafortes das planicies do Piemonte, porque Colômbia tem uma grande diversidade de frutas exóticas com alto teor nutricional, espécies nativas como a mencionada têm sido considerados e priorizadas como alternativas nas investigações de CORPOICA como uma das frutas tropicais com alto potencial de desenvolvimento agrícola, embora na Colômbia apenas se registre nos 
departamentos de Amazonas e Vaupés, por esta razão, é importante rever a informação gerada sobre a espécie e analisar o potencial deste como um sistema produtivo para as condições dos contrafortes das planicies do Piemonte. A espécie é importante por seu alto valor nutricional, superando em aproximadamente 1.5 vezes o acerola (Malpighiae marginata) $(1790 \mathrm{mg} / 100 \mathrm{~g}$ ), em 13 vezes ao marañon (Anacardeum occidentale) $(219 \mathrm{mg} / 100 \mathrm{~g})$ e em 5 vezes o limão (Citrus limon) $(44,2 \mathrm{mg} / 100 \mathrm{~g})$, em comparação com a laranja (Citrus sinensis) contém 30 vezes mais vitamina $C, 10$ vezes mais ferro, 3 vezes mais niacina, 2 vezes mais de riboflavina e $50 \%$ mais de fósforo.

Palavras-chave: Frutas, vitaminas, colheitas, árvores, alimentos.

\section{INTRODUCCIÓN}

El camu camu (Myrciaria dubia) es una planta originaria de la Amazonía donde crece en forma silvestre, con un amplio rango de adaptación a diferentes condiciones agroecológicas entre las que se destaca las del piedemonte llanero Colombiano, porque crece a temperatura máxima de $28-35^{\circ} \mathrm{C}$ y mínima de 17 $22^{\circ} \mathrm{C}$; requiere precipitaciones de $2800 \mathrm{~mm} / \mathrm{año}$ y humedad relativa que oscile entre 78 y $82 \%$ (Justi et al., 2000). El camu camu se presenta como una importante alternativa para establecer sistemas productivos en esta zona de piedemonte, se muestra como una especie promisoria para sobrellevar la coyuntura actual del sector agrícola, donde los cultivos tradicionales como el arroz, el maíz y la soya se han visto afectados por los altos costos de producción y las importaciones (Arias, 2009), lo que demanda la necesidad de estrategias de diversificación de los sistemas productivos. Por esta razón es necesario evaluar las características de esta especie, su ecología, los aspectos técnicos agronómicos y las alternativas de producción. Es imprescindible recopilar información acerca de las características, morfológicas y fisiológicas, requerimientos nutricionales, nivel de adaptación y alternativas para agroindustria, comercio interno y su exportación. El aprovechamiento de este cultivo en Colombia se limita en la mayoría de los casos a la recolección del fruto que realizan grupos indígenas en la región del Putumayo donde crece naturalmente en 
las zonas inundables de los ríos (Peters y Vásquez, 2006), también hay algunos estudios genéticos y de comportamiento en Caquetá y Meta (Osorio, 2001; Rojas et al., 2008).

En este artículo se presentan los aspectos técnicos-agronómicos de la especie y su ecología, además de analizar su potencial como cultivo comercial, teniendo en cuenta su demanda agroecológica y exigencias agronómicas para establecerla como sistema productivo en condiciones del piedemonte llanero, puesto que Colombia tiene gran diversidad de frutas exóticas con alto contenido nutricional, especies nativas como el camu camu han sido consideradas y priorizadas como alternativas en investigaciones con alto potencial para el desarrollo agrícola, por lo tanto es importante revisar la información generada sobre la especie.

\section{ORIGEN Y DISTRIBUCIÓN}

El camu camu es una especie nativa de la cuenca del Amazonas occidental, distribuida ampliamente en Perú, Colombia, Brasil y Venezuela. En Colombia se encuentran poblaciones naturales en los departamentos de Amazonas y Putumayo en las márgenes del río Putumayo (Hernández y Barrera, 2010).

El camu camu crece de manera natural en las orillas de los ríos de la Amazonía, indicando que la mayor concentración de poblaciones y de diversidad se encuentra en la Amazonía peruana (Imán et al., 2011), se encuentra a lo largo del río Amazonas hasta el estado de Amazonas en Brasil, así como en la cuenca superior del río Orinoco, y en el estado de Rondonia, Brasil (Peters y Vásquez, 2006). En Colombia, se registra en el departamento de Amazonas en localidades de Amacayacú y Tarapacá; y en el Vaupés en Tabú sobre el río Vaupés (Hernández y Barrera, 2010).

\section{CARACTERÍSTICAS MORFOLÓGICAS}

La planta es un arbusto que alcanza $4 \mathrm{~m}$ de altura, con una copa frondosa, irregular, con ramas delgadas flexibles y pendientes, el tallo es delgado con un diámetro de hasta $15 \mathrm{~cm}$ muy ramificado; el tallo y las ramas son glabros, 
cilíndricos, lisos, de color marrón claro o rojizo y con corteza que se desprende de forma natural; también existe variabilidad morfológica, encontrándose plantas de tipo columnar u ortotrópica ramificándose a partir de 50 a $70 \mathrm{~cm}$ de la base del tallo y el tipo cónica o plagiotrópica (Imán et al., 2011).

Osorio, (2001) asegura que la especie presenta raíces adventicias en el tallo y en las ramas, las cuales se desarrollan altamente en las zonas bajas sometidas a crecientes e inundaciones de las quebradas y ríos, las raíces pueden alcanzar 50 cm de longitud y están adaptadas para soportar la excesiva humedad; las hojas son simples, opuestas, peciolo cilíndrico de $5 \mathrm{~mm}$ de largo, limbo de 4 a $8 \mathrm{~cm}$ de longitud, de 3 a $4 \mathrm{~cm}$ de ancho, lanceolado, borde revoluto, membranáceo; haz de color verde lustroso y envés verde claro.

Los botones florales nacen en grupos en las axilas de las hojas de preferencia de las ramas mayores y también del tronco principal, en una misma yema pueden encontrarse desde 1 a 25 botones, flores hermafroditas, cáliz, corola con pétalos de color blanco que luego de la fecundación se tornan de color marrón (Imán et al., 2011). Las flores son simples, tamaño mediano, cáliz con cinco sépalos soldados, libres, blanquecinos, de prefloración imbricada, descendente; estambres numerosos, finos, alargados y blanquecinos; ovario ínfero (Osorio, 2001).

El fruto de camu camu es una baya globular con $3 \mathrm{~cm}$ de diámetro, cáscara de color negra violácea, pulpa jugosa, blanquecina o teñida de rosa, con suaves fibrillas, la cual es ligeramente ácida, aromática; con 2 a 4 semillas, excepcionalmente con una, tienen en promedio $1.48 \mathrm{~cm}$ de largo, $1.13 \mathrm{~cm}$ de ancho, $0.52 \mathrm{~cm}$ de grosor y un peso de 0.74 gramos (Osorio, 2001). En cuanto al color del fruto, este varía según el estado de maduración que va de verde a rojo, alcanzando el 100\% de coloración rojiza (Imán et al., 2011).

\section{BIOLOGÍA FLORAL}

La floración generalmente empieza cuando la planta alcanza un diámetro basal de $2 \mathrm{~cm}$, la cual no está sincronizada en cada planta, puesto que ocurren varios ciclos durante el año. Las yemas florales se producen primero en la parte distal de las 
ramas más altas, y después que éstas han abierto y ha pasado la polinización, otras yemas salen de un lugar más próximo sobre la rama, por lo tanto, una planta puede presentar simultáneamente yemas florales, flores y frutos en varios estados de desarrollo (Figura 1). En cada nudo se observan hasta 12 flores; también se presenta formación de flores directamente en el tronco y en las ramas gruesas de los individuos grandes (Peters y Vásquez, 2006).

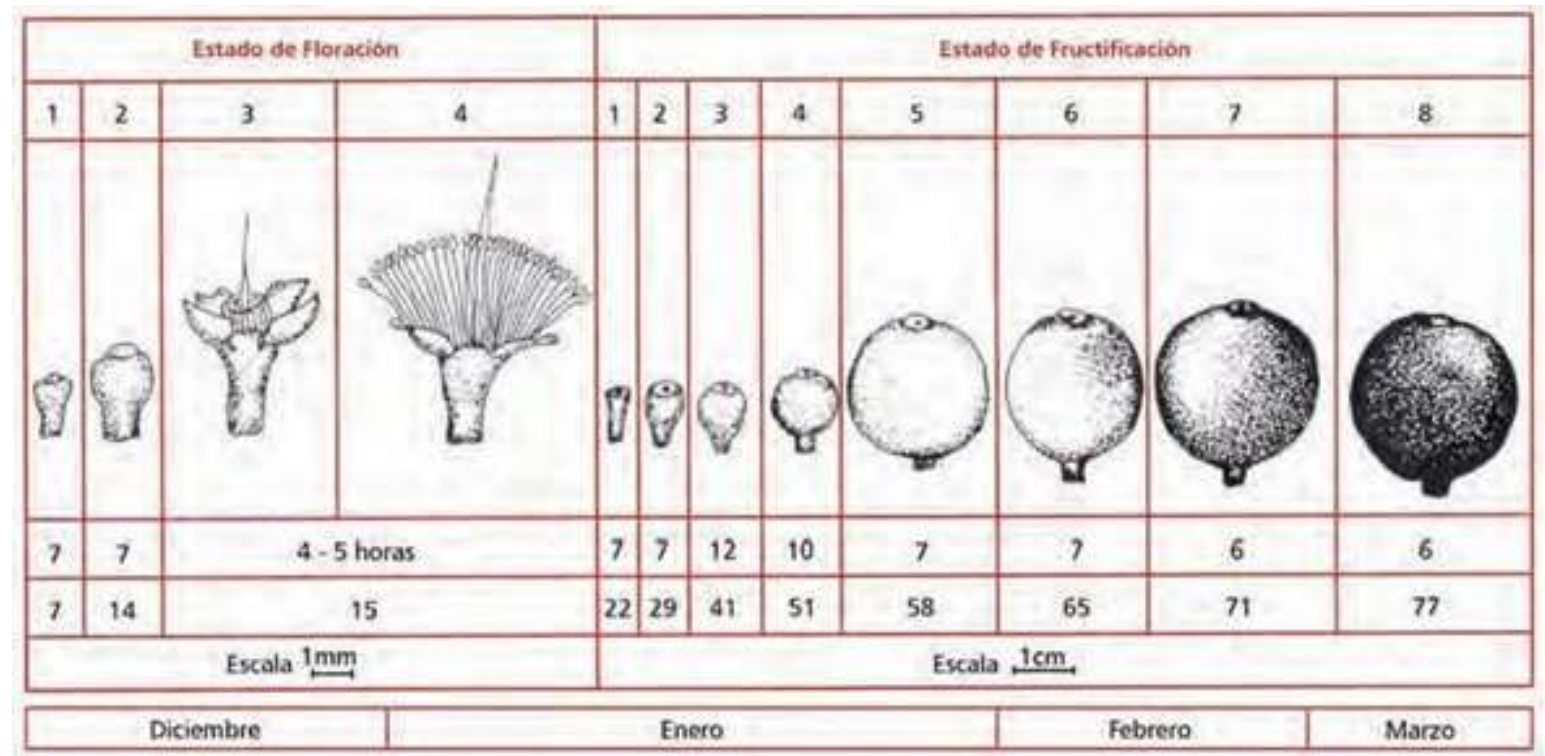

Figura 1. Descripción morfológica de la fenología de M. dubia.

Fuente: Inga et al., (2001).

Cuando las flores están en forma individual son hermafroditas, la antesis ocurre temprano en la mañana siendo receptibles a la polinización por un período de cuatro a cinco horas; una vez sucede esto los estambres empiezan a marchitarse y la corola se seca y cae al día siguiente (Peters y Vásquez, 2006). La emergencia del estilo y los estambres dentro de una flor demuestra un protógino muy marcado; durante la antesis, el estilo sale primero y después los estambres. Peters y Vásquez, (2006) indican que este mecanismo es muy efectivo para evitar la autogamia; aparentemente en el momento que emergen los estambres para liberar polen, el estigma ya no está receptible a la polinización. Aunque $M$. dubia muestra dicogamia, no se descarta la posibilidad de autofecundación por 
geitonogamia debida a la falta de sincronía floral, puesto que el polen de otras flores sobre la misma planta puede efectuar hasta $91 \%$ de polinización.

Peters y Vásquez, (2006) concluyen que el camu camu presenta alogamia facultativa pero no obligatoria, no se observa incompatibilidad genética, aunque parte de la polinización puede ser efectuada por el viento, otros polinizadores de la especie son pequeñas abejas. Las flores contienen néctares y exudan una fragancia dulce y agradable, por lo que en la mañana están cubiertas por abejas como Melipona fuscopilara y Trigona portica.

\section{CARACTERÍSTICAS FÍSICO QUÍMICAS DEL FRUTO}

El fruto de camu camu tiene un buen porcentaje de pulpa aprovechable (Figura 2). El fruto es de forma globular, el color de su cascara varía con el estado de maduración, son verdes con menos del $25 \%$ de coloración rojiza; pintones 25 a $75 \%$ y maduros $100 \%$ de coloración rojiza. Estudios realizados a germoplasma de la especie reportan que el tamaño del fruto está ligado al peso; se consideran frutos pequeños con diámetro menores y peso menores a $2.5 \mathrm{~cm}$ y $9 \mathrm{~g}$ respectivamente, medianos diámetro entre 2.5 y $3 \mathrm{~cm}$, y peso entre 9 y $13 \mathrm{~g}$, frutos grandes para aquellos que miden más de $3 \mathrm{~cm}$ de diámetro y peso mayor a $13 \mathrm{~g}$ (Imán et al., 2011).

\section{Composición del fruto de camucamu}

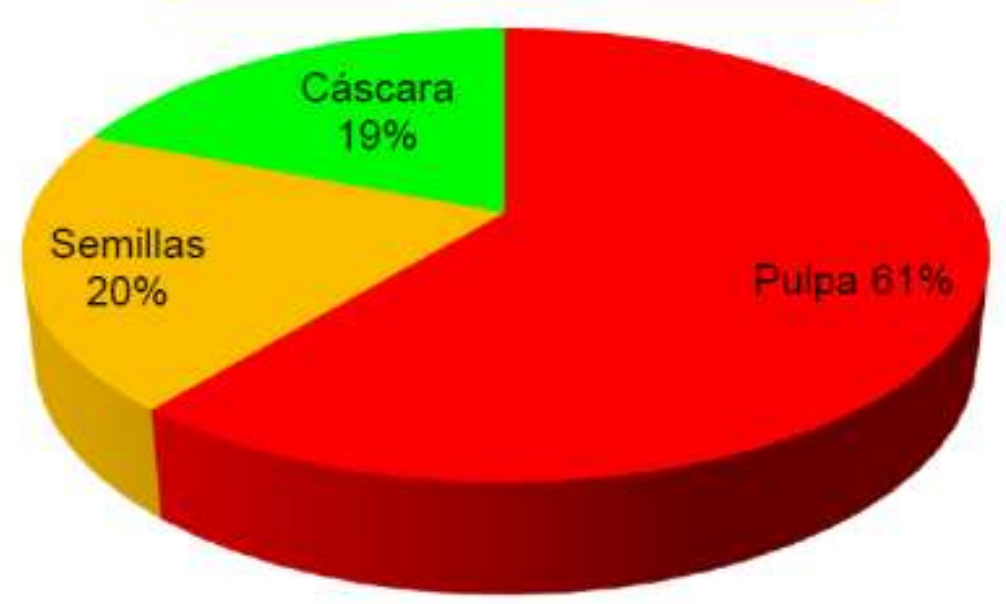

Figura 2. Composición del fruto de camu camu. Fuente: Osorio, (2001). 
Este fruto se destaca el alto contenido de ácido ascórbico en la pulpa (Tabla 1), alcanzando más de $2700 \mathrm{mg}$ en $100 \mathrm{~g}$ de pulpa, siendo su concentración superior a la reportada en otras frutas tropicales. Por su alto contenido de vitaminas el fruto de camu camu ha sido reconocido como un alimento de excelente calidad nutricional (Pinedo y Paredes, 2011), porque supera en 1.5 veces el contenido de vitamina C a la acerola (Malpighiae marginata) $(1790 \mathrm{mg} / 100 \mathrm{~g})$, en 13 veces al marañon (Anacardeum occidentale) $(219 \mathrm{mg} / 100 \mathrm{~g})$ y en 5 veces al limón (Citrus limon) (44.2 mg/100 g), en comparación con la naranja (Citrus sinensis), el fruto del camu camu provee 30 veces más vitamina $C, 10$ veces más hierro, 3 veces más niacina, 2 veces más riboflavina y $50 \%$ más fósforo (Imán et al., 2011).

Tabla 1. Valor nutricional de $100 \mathrm{~g}$ de pulpa de camu camu.

\begin{tabular}{ccc}
\hline Componente & Unidad & Valor \\
\hline Agua & $\mathrm{G}$ & 94.4 \\
Valor energético & $\mathrm{Cal}$ & 17.0 \\
Proteínas & $\mathrm{G}$ & 0.5 \\
Carbohidratos & $\mathrm{G}$ & 47 \\
Fibra & $\mathrm{G}$ & 0.6 \\
Ceniza & $\mathrm{G}$ & 0.2 \\
Calcio & $\mathrm{Mg}$ & 27.0 \\
Fósforo & $\mathrm{Mg}$ & 17.0 \\
Hierro & $\mathrm{Mg}$ & 0.5 \\
Tiamina & $\mathrm{Mg}$ & 0.01 \\
Riboflamina & $\mathrm{Mg}$ & 0.04 \\
Niacina & $\mathrm{Mg}$ & 0.062 \\
Ácido ascórbico reducido & $\mathrm{Mg}$ & 2.780 .0 \\
Ácido Ascórbico Total & $\mathrm{Mg}$ & 2.994 .0 \\
\hline
\end{tabular}

Fuente: Villachica. (1996)

\section{IMPORTANCIA SOCIOECONÓMICA Y AGROINDUSTRIAL}

La mayor concentración del camu camu se encuentra en la Amazonía peruana y brasileña, siendo estos los únicos países que actualmente exportan productos en 
base a esta fruta, en menor escala se le encuentra en Colombia, Venezuela y Bolivia. La producción, recolección y comercialización constituye una importante actividad económica y ecológica en la Amazonía, puesto que genera ocupación permanente del campesino ribereño (pescador, recolector y agricultor estacional) y disminuye la presión que podría producir en los bosques primarios (Peters y Vásquez, 2006). En Colombia, el Instituto Amazónico de Investigaciones Científicas (SINCHI), reporta el desarrollo de programas con el apoyo del estado, en los cuales se involucran las comunidades indígenas y población ribereña de los ríos donde se recolecta la fruta que crece de manera natural, principalmente en el departamento del Putumayo, actualmente varias familias se beneficiaban económicamente de dicha actividad (Hernández y Barrera, 2010).

El camu camu debe ser valorado como producto ancestral y una parte de la biodiversidad de varios países entre ellos Colombia, por su sabor ácido y exótico puede ser materia prima para la elaboración de diferentes productos para la industria alimenticia, puesto que con su pulpa se pueden preparar salsas, mermeladas, jugos, helados, néctares y concentrados (Osorio, 2001).

Por su alto contenido de vitamina $\mathrm{C}$, antioxidantes, potasio y antocianinas, elementos importantes en la salud humana, la pulpa de camu camu puede ser utilizada para la producción de medicinas de origen natural (Muñoz et al., 2007), siendo el ácido ascórbico, un importante antioxidante que ayuda en la prevención de cáncer, enfermedades del corazón, estrés y es energético; también es fundamental para gametogénesis, síntesis de proteínas en cartílagos, piel y sistema circulatorio, además contribuye al mantenimiento del sistema inmunológico y facilita la absorción de nutrientes, incluyendo el hierro (Alvis et al., 2010). Castro et al., (2013) han observado que esta fruta puede ayudar a la prevención del cáncer, el alzhéimer y a combatir el estrés por su alto nivel de antioxidantes, potasio y antocianinas. En la actualidad en países industrializados se producen capsulas de $1 \mathrm{~g}$ de vitamina $\mathrm{C}$ y la pulpa de camu camu puede ser usada como materia prima para este propósito mediante su liofilización (Vega, 2006). 


\section{EL CAMU CAMU (Myrciaria dubia) EN COLOMBIA}

Esta fruta nativa de la amazonia puede ser importante para impulsar el desarrollo rural, agroindustrial y agro exportador, en las regiones donde se adapta y crece naturalmente (Solís, 2015). A partir del 2004 cuando un grupo de mujeres líderes indígenas del municipio de Tarapaca (Amazonas) crearon una empresa para procesar y comercializar la pulpa de camu camu a nivel nacional, para el disfrute de todo el país. Las comunidades indígenas cosechan la fruta a mano, desplazándose en canoas en zonas inundadas donde los árboles forman plantaciones naturales (Evans, 2013).

Una alianza llamada Amazonas 2030 ubicada en Leticia, promueve el desarrollo de la Amazonía colombiana a través del monitoreo sistemático y periódico de los cambios en la calidad de vida, indicadores socio-ambientales y la sostenibilidad de la región, ha fijado su atención sobre esta especie nativa que entre Marzo y Abril comienza a florecer de manera silvestre, cerca de algunos lagos amazónicos; siendo este fruto la esperanza de progreso para las familias de Tarapacá (Amazonas) quienes pretenden mejorar su calidad de vida a través de la producción de mermeladas, pulpas deshidratadas, néctares y concentrados. 47 personas se dedican a la recolección del fruto en las zonas inundables de los lagos de Tarapacá, donde crece el fruto de forma natural (AMAZONAS-2030, s.f).

La dirección general del Instituto Amazónico de Investigaciones Científicas SINCHI, ejecuta un proyecto de aprovechamiento de la pulpa de camu camu en un Acuerdo para la Prosperidad, realizado en Leticia, además asegura que esta especie es importante y por tanto se debe promover su consumo y comercialización para beneficio económico. Para el procesamiento de pulpa de camu camu, en Tarapacá se cuenta con el registro sanitario del Invima y con el permiso de Corpoamazonia (Corporación para el Desarrollo Sostenible del Sur de la Amazonia), sobre la base de estudios de manejo de la especie, también se ha hecho un importante esfuerzo en la innovación y transferencia de la tecnología, hacia las mujeres, quienes quedaron con un centro de acopio dotado con equipos básicos agroindustriales; a través de este proyecto, se cuenta con una línea de 
trabajo que promueve el uso de la biodiversidad desde el punto de vista sostenible y sustentable por parte de las comunidades (SINCHI, s.f) y se proyecta la exportación de sus productos hacia Europa.

\section{HÁBITAT DE LA ESPECIE}

Villachica, (1996) asegura que a pesar de ser una especie nativa de las zonas aluviales inundables temporales, el camu camu prospera bien en los suelos con buen drenaje, siempre y cuando haya un adecuado suministro hídrico y también crece bien en condiciones de mal drenaje (Figura 3).

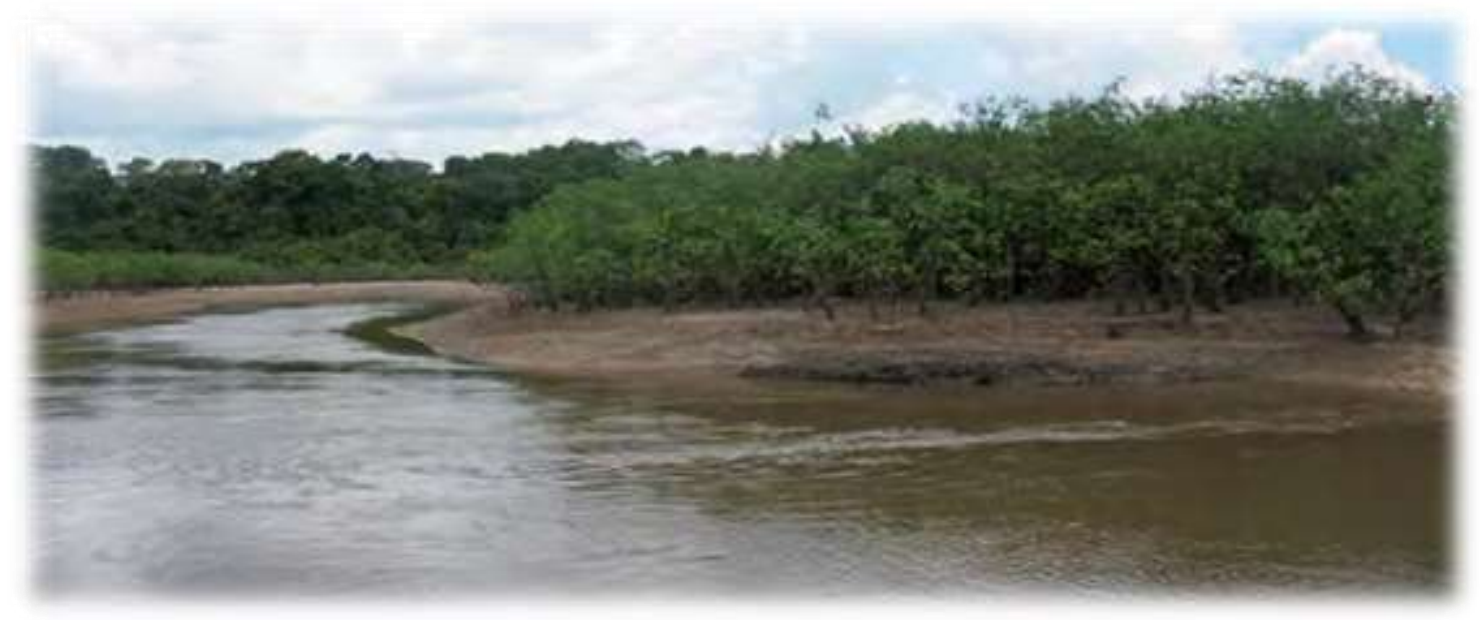

Figura 3. Hábitat de Myrciaria dubia. Fuente: Hernández y Barrera, (2010)

En cuanto a la altitud, se reporta que se adapta bien a alturas desde el nivel del mar hasta 800 msnm, con una radiación solar de 1300 a 2000 horas/año (Osorio, 2001).

\section{SISTEMAS DE PROPAGACIÓN DE LA ESPECIE}

La especie se propaga normalmente por semilla, la cual se debe obtener de plantas sanas, vigorosas y con frutos de buen tamaño; puede tener un porcentaje de germinación cercano al 100\%, la cual inicia aproximadamente a los 12 días después de la siembra, estando aptas para el trasplante a los 270 días de edad, tiempo tras el cual la planta alcanza un tamaño promedio de $40 \mathrm{~cm}$; el trasplante al sitio definitivo debe hacerse a los 30 días después del trasplante a bolsa, cuando la planta tiene un tamaño mínimo de $15 \mathrm{~cm}$ y 7 hojas (Osorio, 2001). 
El rendimiento del camu camu puede mejorarse a través de la injertación, se recomienda utilizar semilla de árboles obtenidos por reproducción sexual y los frutos deben ser cosechados maduros y de color violeta, actividad que principalmente se realiza entre los meses de Diciembre y Marzo. Se recomienda obtener las semillas de las árboles que produzcan más de $15 \mathrm{~kg}$ de fruta por planta con buen vigor y que estén libres de plagas y enfermedades, porque estas plantas producirán portainjertos más vigorosos, pues los mismos árboles de camu camu cumplen esta función (Enciso, 1992).

Las semillas limpias y seleccionadas deben ser colocadas en tratamientos de pregerminado, lavándolas y orándolas para posteriormente colocarlas dentro de bolsas de polietileno transparente (Chagas et al., 2012); la germinación se inicia a los 20 días, lográndose también altos porcentajes; este método permite manipular mayor cantidad, sin necesidad de utilizar abundante sustrato, ni riegos adicionales. La desventaja es que las semillas germinadas deben ser trasladadas al almácigo antes que desarrollen raíces de $3 \mathrm{~cm}$ de longitud, caso contrario tienden a etiolarse fácilmente (Enciso y Villachica, 1993).

La cama de almácigo comúnmente utilizada para el camu camu tiene un desnivel (10 cm debajo del nivel del suelo), el cual permite retener humedad después de cada lluvia o riego favoreciendo el desarrollo de las plantas, que logran $0.7 \mathrm{~m}$ de altura en seis meses (Chagas et al., 2012). En el terreno para almácigo se deben eliminar las malezas y todo el material grueso, luego trazar las camas de manera intercalada con caminos que serán utilizados para el desplazamiento del personal. El ancho de las camas es de un metro, con el largo de $10 \mathrm{~m}$, y la profundidad de $40 \mathrm{~cm}$, quedando las calles para personal con $50 \mathrm{~cm}$ de ancho; una vez preparadas las camas a desnivel, se agregan 40 a $50 \mathrm{~kg}$ de gallinaza más $10 \mathrm{~kg}$ de superfosfato triple por cada $10 \mathrm{~m}^{2}$, lo cual se mezcla hasta una profundidad de $30 \mathrm{~cm}$, esto se puede realizar con facilidad cuando el suelo está ligeramente húmedo, empleando una pala recta o un trinche, es necesario quitar terrones hasta dejar el sustrato bien mullido y nivelado; a continuación utilizando un pequeño punzón se trazan y efectúan los hoyos en las camas, distanciados a 10 
cm entre plantas y $10 \mathrm{~cm}$ entre hileras, en cada hoyo se coloca una semilla pregerminada de camu camu, cubriendo esta semilla con un cm de tierra (Enciso, 1992).

Las plántulas deben permanecer en el almácigo a desnivel por lo menos seis meses, generalmente hasta lograr una altura promedio de $80 \mathrm{~cm}$ y un diámetro del tallo entre 6 y $9 \mathrm{~mm}$ a $30 \mathrm{~cm}$ del suelo. En estas condiciones las plántulas están listas para ser injertadas (Enciso y Villachica, 1993). Todos los brotes que emerjan del tallo principal por debajo de $40 \mathrm{~cm}$ deberán ser eliminados porque el injerto se realizará a $30 \mathrm{~cm}$ sobre el nivel del suelo (Suguino, 2002). Para la cama de injertación se prefieren los suelos de textura franco arcillosa, por su mayor retención de agua, se deben emplear sólo aquellas plantas que muestren buen vigor, es decir aquellas que hayan logrado altura y diámetro superior a $70 \mathrm{~cm}$ y 7 $\mathrm{mm}$ respectivamente; la distancia de siembra es $60 \mathrm{~cm}$ entre hileras y $40 \mathrm{~cm}$ entre plántulas, con hoyos de $25 \mathrm{~cm}$ de profundidad y diámetro de alrededor de $15 \mathrm{~cm}$. El trasplante debe efectuarse durante la época de lluvias, en caso debe contarse con riego, para asegurar mayor porcentaje de prendimiento. Las plantas de camu camu permanecerán allí diez meses hasta su trasplante al campo definitivo: dos meses desde el trasplante del almácigo hasta el momento del injerto y ocho más desde el injerto hasta el trasplante a campo definitivo, este período es requerido para lograr plantas con buen vigor para el trasplante (Enciso y Villachica, 1993).

La época más apropiada para el injerto del camu camu son los meses que corresponden a la época de mayor precipitación, porque existe buena humedad en el suelo para el transporte de sustancias en la planta y permite la cicatrización rápida de las heridas. El diámetro adecuado del tallo del patrón para realizar el injerto está entre 6 y $9 \mathrm{~mm}$ de grosor a $30 \mathrm{~cm}$ de altura sobre el suelo. Las plantas deben tener 0.7 a $1 \mathrm{~m}$ de altura, respectivamente a esos diámetros. Las yemas a emplearse para el injerto deberán provenir de ramas del año, de plantas adultas seleccionadas por sus buenas características. El método de injerto usado es el de astilla (Enciso, 1992), que consiste en realizar un primer corte que penetre en el portainjerto una cuarta parte del grosor del mismo, luego aproximadamente a $2 \mathrm{~cm}$ 
más arriba se hace un segundo corte hacia abajo, hasta que conecte con el primero; después se coloca la yema con astilla en el portainjerto, y luego se realiza el amarre con la cinta plástica cubriendo toda la yema, el cual deberá permanecer durante 60 días hasta que las heridas de los cortes cicatricen bien. Después se procede con el corte a bisel del patrón 5 a $10 \mathrm{~mm}$ sobre el lugar del injerto, con la finalidad de estimular el brote y desarrollo de la yema del injerto (Enciso y Villachica, 1993).

El portainjerto empieza a emitir nuevos brotes debajo o cerca del injerto a los 10 días, siendo variable el número de brotes emitidos, según el vigor del patrón, pero todos ellos deben ser eliminados en forma continua, realizando estas podas hasta que el injerto logre la dominancia apical, momento en el que el patrón disminuirá la intensidad de emisión de brotes, lo cual favorece el desarrollo del injerto. Tan pronto el brote del injerto alcance $20 \mathrm{~cm}$ de longitud será conveniente colocar tutores para evitar su ruptura y facilitar el crecimiento recto, los cuales se deben mantener durante dos a tres meses hasta que el injerto pueda sostenerse por sí solo. Una vez que el injerto tenga un diámetro similar al patrón en la zona de unión, estará listo para ser llevado al campo definitivo. Las plantas injertadas pueden ser podadas a $30-40 \mathrm{~cm}$ sobre el injerto, con el objetivo de inducir la ramificación de la nueva planta e iniciar la formación de la copa a partir de esta altura (Enciso y Villachica, 1993).

\section{REQUERIMIENTOS AGRONÓMICOS PARA EL CULTIVO DE CAMU CAMU}

Una vez seleccionado y muestreado el terreno, el área a sembrar deberá ser marcada de acuerdo con las distancias escogidas, haciendo hoyos de acuerdo con el tamaño de la planta a trasplantar, generalmente con un diámetro de $30 \mathrm{~cm}$, y una profundidad de 30 a $40 \mathrm{~cm}$. En suelos ácidos con más de $50 \%$ de saturación con aluminio se sugiere la aplicación de 50 a $100 \mathrm{~g}$ de cal molida y 50 a $100 \mathrm{~g}$ de roca fosfatada, al fondo del hoyo donde se ubicará la planta, las dos enmiendas deben aplicarse 15 días antes de sembrar el camu camu y ser cubiertas con $3 \mathrm{~cm}$ de tierra para que las raíces de la planta no entren en contacto directo con ellas (Enciso, 1992). 
Aunque la distancia de siembra depende de varios factores, entre los cuales están la fertilidad del suelo, el nivel tecnológico, calidad genética y si esta se realiza en monocultivo o en asocio con maíz, yuca, arroz, caña de azúcar y bijao (Pinedo et al., 2010); se sugiere que la distancia de siembra sea de cuatro metros entre hileras y tres entre plantas, para permitir una mayor eficiencia en el uso de la radiación solar cuando la plantación haya alcanzado su desarrollo pleno, además de facilitar la siembra de cultivos asociados y el uso de mecanización agrícola (Figura 4).
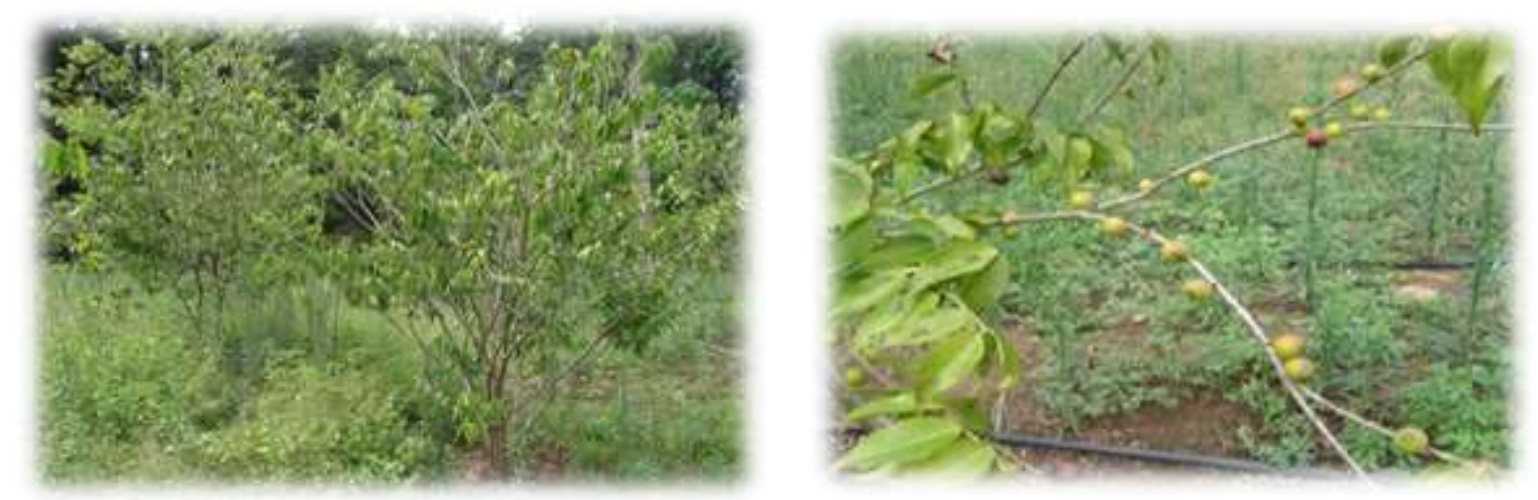

Figura 4. Plantas de camu camu en un sitio definitivo en estado vegetativo (izquierda) y fructificada (derecha). Fotografías tomadas por Leonardo Ardila en CORPOICA la Libertad, (2013)

Es muy importante desde la etapa del almácigo empezar con la aplicación de los fertilizantes cuando las plántulas tengan $20 \mathrm{~cm}$ de altura, utilizando $50 \mathrm{~g}$ de urea más $25 \mathrm{~g}$ de cloruro de potasio por $\mathrm{m}^{2}$, realizando tres fertilizaciones durante los seis meses que dura esta etapa; si se desea efectuar fertilización foliar, se puede realizar con urea (5 g/20 litros de agua) Villachica, (1996). En estudios realizados en la localidad de Pucallpa en Perú, en donde se analizaron muestras foliares con síntomas de deficiencia nutricional, haciendo la comparación con hojas normales, se encontraron niveles bajos de fósforo, potasio, nitrógeno, boro, magnesio y zinc (Tabla 3), aunque no se han realizado estudios sobre los requerimientos nutricionales del camu camu, se ha observado que es más susceptible a las deficiencias de fósforo y potasio cuando es cultivado en suelos ácidos con deficiencias de estos nutrimentos (Riva, 1994). La fertilización debe ser efectuada 
en base a los resultados del análisis de suelo, además tener en cuenta que la fertilización con roca fosfatada y cal deben efectuarse al fondo del hoyo, por lo menos un mes antes del trasplante; los abonos de mantenimiento (nitrógeno, potasio y magnesio) se colocan en la prolongación de la copa, en una circunferencia de $5 \mathrm{~cm}$ de ancho por $5 \mathrm{~cm}$ de profundidad y se debe cubrir con tierra para evitar la volatilización del nitrógeno o la pérdida por escorrentía. Los abonos de mantenimiento se aplican antes de la floración y al cuajado de los frutos, lo cual disminuye las pérdidas por lixiviación y aumenta la eficiencia en el uso de nutrientes, sin embargo, el número de aplicaciones anuales que se efectúen depende de la disponibilidad de mano de obra para realizar esta actividad.

Tabla 3. Nivel de nutrientes en hojas jóvenes de camu camu normales y en hojas con deficiencias nutricionales

\begin{tabular}{ccccc}
\hline Nutriente & Unid & Hoja normal & Hoja deficiente & Def/Norm \\
\hline $\mathrm{N}$ & $\%$ & 3.16 & 2.49 & 0.79 \\
$\mathrm{P}$ & $\%$ & 0.27 & 0.12 & 0.44 \\
$\mathrm{~K}$ & $\%$ & 1.03 & 0.44 & 0.43 \\
$\mathrm{Ca}$ & $\%$ & 0.50 & 0.48 & 0.96 \\
$\mathrm{Mg}$ & $\%$ & 0.18 & 0.15 & 0.83 \\
$\mathrm{Fe}$ & $\mathrm{ppm}$ & 98 & 97 & 0.99 \\
$\mathrm{Zn}$ & $\mathrm{ppm}$ & 47 & 39 & 0.83 \\
$\mathrm{Ca}$ & $\mathrm{ppm}$ & 9 & 8 & 0.89 \\
$\mathrm{Mn}$ & $\mathrm{ppm}$ & 764 & 868 & 1.14 \\
$\mathrm{~B}$ & $\mathrm{ppm}$ & 90 & 68 & 0.76 \\
\hline
\end{tabular}

Fuente: Riva, (1994).

El manejo de malezas está relacionado con la edad y desarrollo del cultivo, el cual puede realizarse manualmente, aplicando herbicidas con medios mecánicos o con la ayuda de coberturas. El control manual se efectúa tres a cuatro veces al año, dependiendo de la intensidad en el crecimiento de las malezas; la demanda de mano de obra disminuye conforme la planta de camu camu desarrolla su copa y produce sombra que inhibe el desarrollo de otras especies. Cuando no se tienen 
cultivos asociados es posible el uso de herbicidas biodegradables o de maquinaria para el control de arvenses, lo cual se logra con el paso de rastras cruzadas o plateo manual alrededor de la planta (Villachica, 1996).

\section{RENDIMIENTO DEL CULTIVO}

Los rendimientos de camu camu observados en plantaciones naturales pueden estimarse a partir de los datos presentados por Villachica, (1996) quien asegura que las plantas maduras con $12 \mathrm{~cm}$ de diámetro de tallo o más, rinden cerca de 30 $\mathrm{kg}$ de fruta cada una, asumiendo una copa de $3 \mathrm{~m}$ de diámetro, se tendría que la plantación equivalente sería de 625 plantas/ha, lo que resultaría en una producción de 18.44 ton/ha; si la densidad de siembra fuese de 833 plantas/ha, entonces la productividad resultante sería de 15.79 ton/ha. Pinedo, (2009) reporta rendimientos crecientes a través de 15 años de aprovechamiento de la plantación, en la región de Loreto en Perú, la cual inicia su edad productiva a los 3 años, con un rendimiento menor a una tonelada por hectárea; a los 8 años se incrementa a 9 toneladas y durante 15 años alcanza más de 28 toneladas por hectárea, lo cual se da porque el diámetro de su tallo se engrosa con la edad (Flores y Miranda, 2017) (Tabla 4).

Tabla 4. Rendimiento potencial de fruta de camu camu en función al diámetro del tallo de la planta

\begin{tabular}{cccc}
\hline Diámetro $(\mathbf{c m})$ & kg/Planta & Plantas/ha & Ton/ha \\
\hline $2-3.9$ & 3.22 & 2400 & 7.73 \\
$4-5.9$ & 4.80 & 1666 & 8.00 \\
$6-7.9$ & 6.55 & 1333 & 8.73 \\
$8-9.9$ & 11.36 & 1111 & 12.62 \\
$10-11.9$ & 18.96 & 833 & 15.79 \\
$12-14.0$ & 29.50 & 625 & 18.44 \\
\hline
\end{tabular}

Fuente: Adaptado de Flores y Miranda, (2017). 


\section{COSECHA Y POSCOSECHA}

En Colombia uno de los territorios donde se aprovecha comercialmente el camu camu es en Tarapacá (Amazonas), Hernández y Barrera, (2010) reportan que la floración de la especie en esta localidad se da a comienzos de Octubre y durante los meses de Diciembre y Enero, por lo cual la cosecha se concentra en los meses de Febrero a Marzo; el botón floral dura 16 días aproximadamente, dando paso al estado de flor abierta que dura 4 días más, posteriormente se presenta engrosamiento de ovario que es cuando los frutos son muy pequeños, estado en el que dura desarrollándose 48 días, tiempo tras el cual se pueden identificar los frutos bien formados de color verde durando 10 días así, dando paso al fruto maduro el cual dura en la planta por un periodo de 10 días más. En total el ciclo completo desde botón floral hasta fruto maduro toma 85 días, empezando a finales de Diciembre y prolongándose hasta mediados de Marzo.

La cosecha en las plantaciones naturales ubicadas en las orillas de los ríos se efectúa utilizando canoas, los frutos se recogen dos veces por semana en la época de mayor producción y cuando empiezan a madurar, es decir cuando están en estado verde sazón (Hernández y Barrera, 2010).

Se reconoce el proceso de maduración porque la cáscara que es de color verde adquiere algunas pintas color rojizo; tres a cuatro días después de colectados los frutos toman un color rojizo intenso; si la fruta va a ser utilizada en la producción de ácido ascórbico, entonces la cosecha debe hacerse en estado verde para mantener su acidez cítrica (Villachica, 1996). Una vez cosechada la fruta debe ser colocada en recipientes de madera o en canastas con capacidad máxima de 10 $\mathrm{kg}$, para evitar el deterioro por aplastamiento, resguardados en la sombra. La fruta menos madura tiene algo más de consistencia, en condiciones de plantaciones naturales se utilizan cajones de madera y canastas de fibra. No se tiene un flujo de proceso definido para el manejo de la fruta después de la cosecha, sin embargo, se sugiere que el transporte al lugar de procesamiento debe realizarse a la brevedad posible, con el fin de que la fruta sea lavada, oreada y seleccionada (Villachica, 1996). 


\section{PROCESO DE INDUSTRIALIZACION DE LA FRUTA}

La fruta de camu camu puede ser empleada para la fabricación de jugos, helados, concentrados, néctares, mermeladas y para la obtención de ácido ascórbico natural; el jugo y los helados de camu camu son producidos y consumidos de manera tradicional en las poblaciones donde se encuentra esta fruta, debido a su alto contenido de ácido ascórbico la pulpa tiene que ser diluida previamente a su consumo. Los concentrados no son preparados todavía, debido a la ausencia de materia prima, que no ha permitido desarrollar extensivamente la tecnología; sin embargo, algunas empresas privadas están efectuando ensayos para producir concentrados tipo pasta diluida o "squash" diluido, en los cuales se mantiene al máximo la vitamina $\mathrm{C}$ natural que posee la fruta. Recientemente se ha iniciado la producción de tabletas de ácido ascórbico natural en base a la extracción de este producto del camu camu; se producen tabletas de polvo deshidratado de camu camu, que contienen $50 \%$ de vitamina $\mathrm{C}$, a las cuales se les agrega algún otro producto naturista para hacerlo más atractivo, como por ejemplo el propóleo producido por las abejas. Las cápsulas de vitamina $C$ se recomiendan en este caso para situaciones de intensa actividad física, entre otros. Las investigaciones en el campo de la medicina han determinado que las personas con alto nivel de vitaminas antioxidantes en la sangre tienen menos posibilidad de desarrollar enfermedades degenerativas (Biocomercio Perú - PROMPEX / SUNAT, 2017).

\section{ANÁLISIS}

El camu camu es una especie resistente a las inundaciones, capaz de permanecer por cerca de cuatro a cinco meses en condiciones de total anegación, esta característica si se analiza teniendo en cuenta la gran cantidad de suelos de vegas, de terrazas bajas y de sabanas inundables que se presentan en el piedemonte llanero, podría ser una alternativa de desarrollo agrícola para estas zonas, sustentada en la generación de conocimiento sobre los requerimientos agroecológicos de la especie en condiciones de piedemonte, tal como se observó en algunos estudios realizados en Caquetá, donde su comportamiento fue bueno. 
En todo el piedemonte llanero la oferta hídrica es alta, en épocas de invierno son reconocidas las pérdidas de los productores agrícolas de cultivos convencionales como plátano, arroz, soya y algodón entre otros, por las inundaciones que se presentan, en este sentido el camu camu se convierte en una buena opción, mitigando las pérdidas económicas y ambientales del productor de la región. Adicionalmente a la oferta ambiental favorable para el desarrollo de este cultivo, es importante reconocer la importancia de la cercanía y facilidad de movilización que tiene el piedemonte llanero en el departamento del Meta, teniendo a Bogotá como el principal centro de acopio del país, lo cual facilita su comercialización, permitiendo que se pueda competir con la oferta proveniente de los departamentos de Amazonas y Putumayo.

Considerando sus condiciones ambientales particulares, es importante desarrollar alternativas productivas que no generen grandes impactos y que por el contrario se articulen de manera sostenible en lo económico, social y ambiental a las condiciones de la zona, en este sentido el reconocimiento de alternativas nativas con potencial de exportación como el camu camu por su gran demanda mundial, se convierte en una excelente opción para el desarrollo agrícola local.

\section{CONCLUSIONES}

La fruta del camu camu, tiene condiciones de producción favorables por su alto contenido de ácido ascórbico, por lo cual es un producto que está ganando importancia a nivel mundial, puesto que son más de 10 países los que actualmente están importando la fruta.

La industria de la pulpa de fruta de camu camu vislumbra un horizonte promisorio por el cuidado de la salud y el creciente interés por el consumo de productos naturales libres de agentes químicos, puesto que el consumidor está dispuesto a pagar un valor agregado por productos de origen natural con alto valor nutritivo.

En Colombia la explotación del camu camu se limita a la recolección de los frutos y algún grado de procesamiento, los departamentos que han incursionado en dichas actividades son Caquetá, Putumayo y Amazonas, este último ha mostrado 
resultados promisorios, con al apoyo del Instituto Amazónico de Investigaciones Científicas ( $\mathrm{SINCHI),} \mathrm{el} \mathrm{cual} \mathrm{ha} \mathrm{liderado} \mathrm{con} \mathrm{éxito} \mathrm{el} \mathrm{establecimiento} \mathrm{de} \mathrm{sistemas}$ productivos con base en la recolección y el procesamiento de la fruta.

En Suramérica, Perú es uno de los mayores exportadores de la fruta a diferentes destinos, las condiciones agroecológicas son de trópico como nuestro país, los adelantos técnicos y tecnológicos de las explotaciones de camu camu en dicho país, pueden ser considerados para las condiciones del piedemonte llanero, puesto que el camu camu es una especie de desarrollo precoz que ha mostrado buena respuesta cuando se tiene en asociación y cuando se establecen sistemas agroforestales, convirtiéndola en una buena alternativa para la diversificación de los sistemas productivos en el piedemonte llanero.

\section{REFERENCIAS BIBLIOGRÁFICAS}

1. Alvis R., Pino J., Gonzáles J., Francia J.C., Shiga B. Efecto citoprotector del camu-camu Myrciaria dubia en tres líneas celulares de ratón expuestos in vivo a bromato de potasio. Revista Peruana de Biología. 17 (3): 389-392. 2010.

2. AMAZONAS-2030, Alianza para la sostenibilidad y la calidad de vida en la Amazonía colombiana. s.f. Recuperado 11 Diciembre 2016. Disponible En: http://www.amazonas2030.net/articulo.html

3. Arias F.J. Perspectivas del agro colombiano frente a la actual crisis financiera. Revista Academia de Economía. (112): 1-8. 2009.

4. Castro J.C., Gutiérrez F., Cinthya A., Cerdeira L.A., Tapullima A., Cobos M., Imán S. Variación del contenido de vitamina $\mathrm{C}$ y antacianinas Myrciaria dubia "camu camu". Rev Soc Quím Perú. 79 (4): 319-330. 2013.

5. Chagas E.A., Bacelar C.G., dos Santos A., Garcia M.I., Tadashi R., Camargo L. Propagação do camu-camu (Myrciaria dubia (HBK) Mcvaugh). Revista Agro@mbiente On-line. 6 (1): 67-73. 2012.

6. Enciso R., Propagación de camu camu (Myrciaria dubia) por injerto. Informe Técnico N. 18. Programa de Investigación en Cultivos Tropicales. INIA, Lima, Perú. 17 p. 1992.

7. Enciso R., Villachica H. Producción y manejo de plantas injertadas de camu camu (Myrciaria dubia) en vivero. Informe Técnico N. 25. INIA, Lima, Perú. 20 p. 1993.

8. Evans T. Una sofisticada fruta llamada Camu Camu. Revista Diners, Bogotá, Colombia. 2013. Recuperado 15 Diciembre 2016. Disponible En: http://revistadiners.com.co/articulo/24 809390 una-sofisticada-fruta-llamadacamu-camu 
9. Flores J., Miranda E. Factores que influyen en la rentabilidad económica de la producción del cultivo de camu camu en la selva peruana. Revista Tzhoecoen. 9 (1): 94-106. 2017.

10. Hernández M.S., Barrera J.A. Camu camu. Instituto Amazónico de Investigaciones Científicas "SINCHI", Bogotá, Colombia. 164 p. 2010.

11. Imán S., Bravo L., Sotero V., Oliva C. Contenido de vitamina $C$ en frutos de camu camu Myrciaria dubia (HBK) Mc Vaugh, en cuatro estados de maduración, procedentes de la Colección de Germoplasma del INIA Loreto, Perú. Scientia Agropecuaria. 2 (3): 123-130. 2011.

12. Inga H., Pinedo M., Delgado C., Linares C., Mejía K. Fenología reproductiva de Myrciaria dubia McVaugh (H.B.K.) camu camu. Folia amazónica. 12 (1-2): 99-106. 2001.

13.Justi K.C., Visentainer J.V., Evelázio d.S.N., Matsushita M. Nutritional composition and vitamin $\mathrm{C}$ stability in stored camu-camu (Myrciaria dubia) pulp. Archivos latinoamericanos de nutrición. 50 (4): 405-408. 2000.

14. Muñoz A.M., Ramos D.F., Alvarado C., Castañeda B. Evaluación de la capacidad antioxidante y contenido de compuestos fenólicos en recursos vegetales promisorios. Revista de la Sociedad Química del Perú. 73 (3): 142149. 2007.

15. Osorio V.E., El cultivo del Camu-Camu Myrciaria dubia HBK Mc. Vaugh: manejo y utilización. Corpoica, Florencia, Colombia. 12 p. 2001.

16. Peters C., Vásquez A. Estudios ecológicos de Camu-Camu (Myrciaria dubia) producción de frutos en poblaciones naturales. Folia amazónica. 1 (1-2): 87102. 2006.

17. Pinedo M. Camu camu innovación del agro en la Amazonia Peruana. Instituto de Investigaciones de la Amazonía Peruana, Loreto, Perú. 55 p. 2009.

18. Pinedo M., Delgado C., Farroñay R., Del Castillo D., Iman S., Villacres J., Fachin L., Oliva C., Abanto C., Bardales R., Vega R. Camu camu (Myrciaria dubia, Myrtaceae); Aportes para su aprovechamiento sostenible en la Amazonía Peruana. Instituto de Investigaciones de la Amazonía Peruana, lquitos, Perú. 135 p. 2010.

19. Pinedo M., Paredes E. Evaluación preliminar de 108 progenies precoces de camu-camu Myrciaria dubia (Myrtaceae) en Loreto, Peru. Folia Amazónica. 20 (1-2): 77-82. 2011.

20. Riva R. Cultivo del camu camu en Pucallpa. Programa de Investigación en Estación Experimental Pucallpa. Instituto de Investigaciones Agropecuarias, Pucalpa, Perú. 19 p. 1994.

21. Rojas S., Rodrigues D., Lima M., Astolfi S. Desenvolvimento e mapeamento de microssatélites gênicos (EST-SSRs) de camu-camu (Myrciaria dubia [HBK] McVaugh). Corpoica Ciencia y Tecnología Agropecuaria. 9 (1): 14-21. 2008.

22. SINCHI, Instituto Amazónico de Investigaciones Científicas. Programa de Fortalecimiento de Emprendimientos del Instituto SINCHI. s.f. Recuperado 12 Diciembre 2016. Disponible En: https://www.sinchi.org.co/negocios/acerca-delproyecto

23. Solís C.C. Modelo de desarrollo para analizar las debilidades, oportunidades, fortalezas y amenazas de la exportación de la fruta camu camu por parte de las empresas colombianas dedicadas a los agro negocios al mercado japonés, 
Administrador de Negocios Internacionales. Escuela de Ciencias Estratégicas, Universidad Pontificia Bolivariana, Medellín, Colombia. 83 p. 2015.

24. Suguino E. Propagação vegetativa do camu-camu (Myrciaria dubia (HBK) McVaugh) por meio da garfagem em diferentes porta-enxertos da família Myrtaceae, Mgister en Agronomia. Escuela Superior de Agricultura, Universidade de São Paulo, Estado de São Paulo, Brasil. 63 p. 2002.

25. SUNAT, PROMPERU, Superintendencia Nacional de Aduanas y de Administración Tributaria y PROMPERU, Comisión de Promoción del Perú para la Exportación y el Turismo. Exportación de producto camu camu según sus principales mercados en kg 2012-2017. 2017. Recuperado 14 Junio, 2017. Disponible http://www.siicex.gob.pe/siicex/apb/ReporteProducto.aspx?psector=1025\&prep orte $=$ prodemprvolu\&pvalor $=1920$

26. Villachica L.H. El cultivo del camu camu (Myrciaria dubia HBK Mc Vaugh) en la Amazonía peruana. Tratado de Cooperación Amazonia, Secretaria ProTempore, 84 p. 1996. 